\title{
The Game Selection Model for the Payoff Strategy Optimization of Mobile CrowdSensing Task
}

\author{
Guosheng Zhao' ${ }^{1}$, Dongmei Liu ${ }^{1 *}$, and Jian Wang ${ }^{2}$ \\ ${ }^{1}$ College of Computer Science and Information Engineering, Harbin Normal University \\ Harbin 150025, China \\ [e-mail: zgswj@163.com, meiliumeidong@163.com] \\ ${ }^{2}$ School of Computer Science and Technology, Harbin University of Science and Technology \\ Harbin 150001, China \\ [e-mail: wangjianlydia@163.com] \\ *Corresponding author: Dongmei Liu
}

Received October 21, 2020; revised January 20, 2021; accepted February 16, 2021; published April 30, 2021

\begin{abstract}
The payoff game between task publishers and users in the mobile crowdsensing environment is a hot topic of research. A optimal payoff selection model based on stochastic evolutionary game is proposed. Firstly, the process of payoff optimization selection is modeled as a task publisher-user stochastic evolutionary game model. Secondly, the low-quality data is identified by the data quality evaluation algorithm, which improves the fitness of perceptual task matching target users, so that task publishers and users can obtain the optimal payoff at the current moment. Finally, by solving the stability strategy and analyzing the stability of the model, the optimal payoff strategy is obtained under different intensity of random interference and different initial state. The simulation results show that, in the aspect of data quality evaluation, compared with BP detection method and SVM detection method, the accuracy of anomaly data detection of the proposed model is improved by $8.1 \%$ and $0.5 \%$ respectively, and the accuracy of data classification is improved by $59.2 \%$ and $32.2 \%$ respectively. In the aspect of the optimal payoff strategy selection, it is verified that the proposed model can reasonably select the payoff strategy.
\end{abstract}

Keywords: Mobile Crowdsensing, Stochastic Evolutionary Game, Data Quality, Credibility, Payoff Selection 


\section{Introduction}

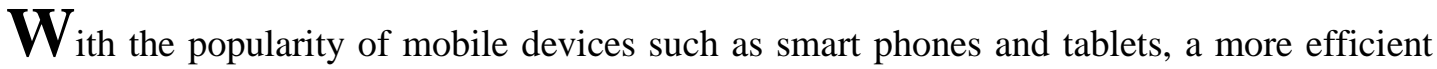
way to collect a large amount of information and data has been brought to the social network of the internet of all things. Mobile crowdsensing(MCS) technology came into being [1]. Mobile crowdsensing refers to the use of mobile devices to collect, analyze and share sensing information and data. MCS has been widely used in various application scenarios, such as environmental monitoring [2], location labeling [3] and traffic flow monitoring [4], etc.

The typical sensing structure of mobile crowdsensing is shown in Fig. 1. MCS consists of three parts: task publishers, users, and cloud platform. In the task release stage: (1) the task publisher sends the task and the incentive mechanism to the cloud platform. (2) The cloud platform publishes the task status information to users. In the sensing data stage: (3) the users use mobile devices to collect sensing data and upload it to the cloud platform. In the payment stage: (4) the cloud platform is responsible for evaluating and screening the data quality submitted by users, and the paying remuneration to users who meet the task requirements. (5) The user's collected sensing data is sent to the task publisher to obtain the task result payoff.

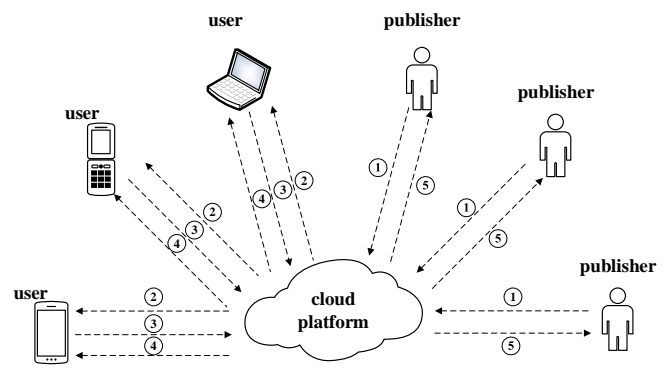

Fig. 1. A typical crowdsensing architecture

So far, the research of task payoff optimization in mobile crowdsensing is mainly focused on the following two aspects: Firstly, the problem of optimal payoff for task publishers and users. Researchers have constructed a variety of mobile crowdsensing payoff models to solve the optimal payoff problem of task publishers and users. But most models are based on complete rationality. For example, Pouryazdan et al. [5] believe that stimulating users in mobile crowdsensing system can improve data value, and propose a mobile intelligent game method from the perspective of data credibility and authenticity to ensure the credibility of users. Nie et al. [6] studied an optimal payoff incentive mechanism for task publishers based on stackelberg game. The mechanism can efficiently recruit a large number of mobile users for data collection, thus increasing the payoff of task publishers. The existing game models considering the bounded rationality of the players are rarely used in MCS. Liu et al. [7] proposed an evolutionary game model to describe the cooperative game phenomenon in mobile crowdsensing network, aiming at the problem that users hope to collect data at low cost and obtain high payoff. The model is a game between users and users. Na et al. [8] put forward the evolutionary game model to ensure the security of the network with the minimum resource cost in order to prevent the network from being attacked by DoS. Secondly, the problem of payoff optimization guided by data quality evaluation. In recent years, the data quality problem of MCS has attracted the attention of many researchers. At present, in order to solve the problem of low-quality data, the existing solutions are mainly divided into incentive strategy and classification strategy. Incentive strategy is to motivate users to provide high-quality data through appropriate incentive measures. Yang et al. [9] proposed a social 
incentive mechanism that motivates participants to submit high-quality data through their credibility in social networks. Peng et al. [10] improved the incentive mechanism from two aspects: data quality and user payoff. The mechanism not only guarantees the data quality, but also improves the overall efficiency. The classification strategy is more focused on the data analysis stage than the incentive strategy. The classification strategy uses the latest machine learning and data mining techniques to estimate the user data quality and filter out abnormal data items, so as to improve the data quality. Yang et al. [11] designed an unsupervised data quality classification method by combining data quality classification with incentive mechanism. The method uses outlier detection technology to filter out abnormal data items. In addition, the surplus sharing process is modeled as a cooperative game, and a method based on shapley value is proposed to determine the reward of each user. Wang et al. [12] proposed a mobile crowdsensing user selection method based on the characteristics of sensing data. The method considers not only the probability of time and space, but also the characteristics of data. The existing methods have advantages, but they also have their own limitations. The incentive strategy only affects the MCS participant selection process, and the classification strategy cannot drive the data source to obtain higher quality data.

However, the above studies ignore two important factors: bounded rationality and learning ability. Because the MCS network topology is constantly changing, the behavior of task publishers and users is a kind of bounded rational behavior. Ignoring the limitation of bounded rationality, modeling the game between the two sides will deviate from the actual situation. The accuracy of the optimal payoff strategy selection method of both sides of the game is reduced. Because evolutionary game has the ability to analyze the game of both sides of bounded rationality, the accuracy can be improved by introducing evolutionary game into the selection of optimal payoff strategy. However, the evolutionary game is also affected by random factors, such as environmental factors, when selecting the optimal payoff strategy. Therefore, we use Itó stochastic differential equation to establish a stochastic evolutionary game model to obtain the optimal payoff strategy and improve the accuracy of the model. Data quality evaluation is also a challenging problem in MCS. Generally speaking, the final payoff of users is directly proportional to the quality of their data [7]. If the data quality of users is not evaluated, it will affect the payoff of high-quality users, thus reducing the effectiveness of mobile crowdsensing. Therefore, under the premise of considering bounded rationality and data quality, this paper models the selection process of payoff strategy as a task publisher-user stochastic evolutionary game model (PUSEGM). The two innovative contributions of this paper are as follows:

(1) A data quality evaluation method is proposed to quantify the user's data quality. By this method, low-quality users and malicious users can be identified. The method improves the fitness of the target users and ensures the payoff of task publishers and legitimate users.

(2) A stochastic evolutionary game model is constructed. The payoff optimization process of task publishers and users is modeled as a stochastic evolutionary game model. And the stability strategy of evolutionary game is deduced.

\section{Model Description}

In mobile crowdsensing, task publishers and users have different payoff strategy selection mechanisms because of their different cognitive levels and skills. Over time, low-payoff task publishers and users will constantly change their strategies to achieve high payoff. This is caused by the traction and learning mechanism of payoff difference. 


\subsection{Model Assumptions}

In the task publisher-user stochastic evolutionary game model, the following assumptions are made for the proposed scheme:

(1) The subject of game is bounded rationality. According to the task requirement, the task publishers choose the incentive strategy to release tasks and gain their payoff. According to the incentive strategy, the users can provide data of different quality levels and get corresponding payoff.

(2) User's data quality strategy and task publisher's incentive strategy change dynamically over time.

(3) In the mobile crowdsensing environment, the payoff of task publishers is directly proportional to the incentive strategy. And the payoff of users is directly proportional to the quality of data provided.

\subsection{Model Construction}

In mobile crowdsensing, the payoffs of task publishers and users are related to the quality of sensing data submitted by users. And different tasks have different concerns about their data. Therefore, this paper proposes a stochastic evolutionary game model which combines data quality evaluation and payoff strategy optimization. Considering the influence of bounded rationality and random factors, the task publisher-user stochastic evolution game model is constructed, as shown in Fig. 2.

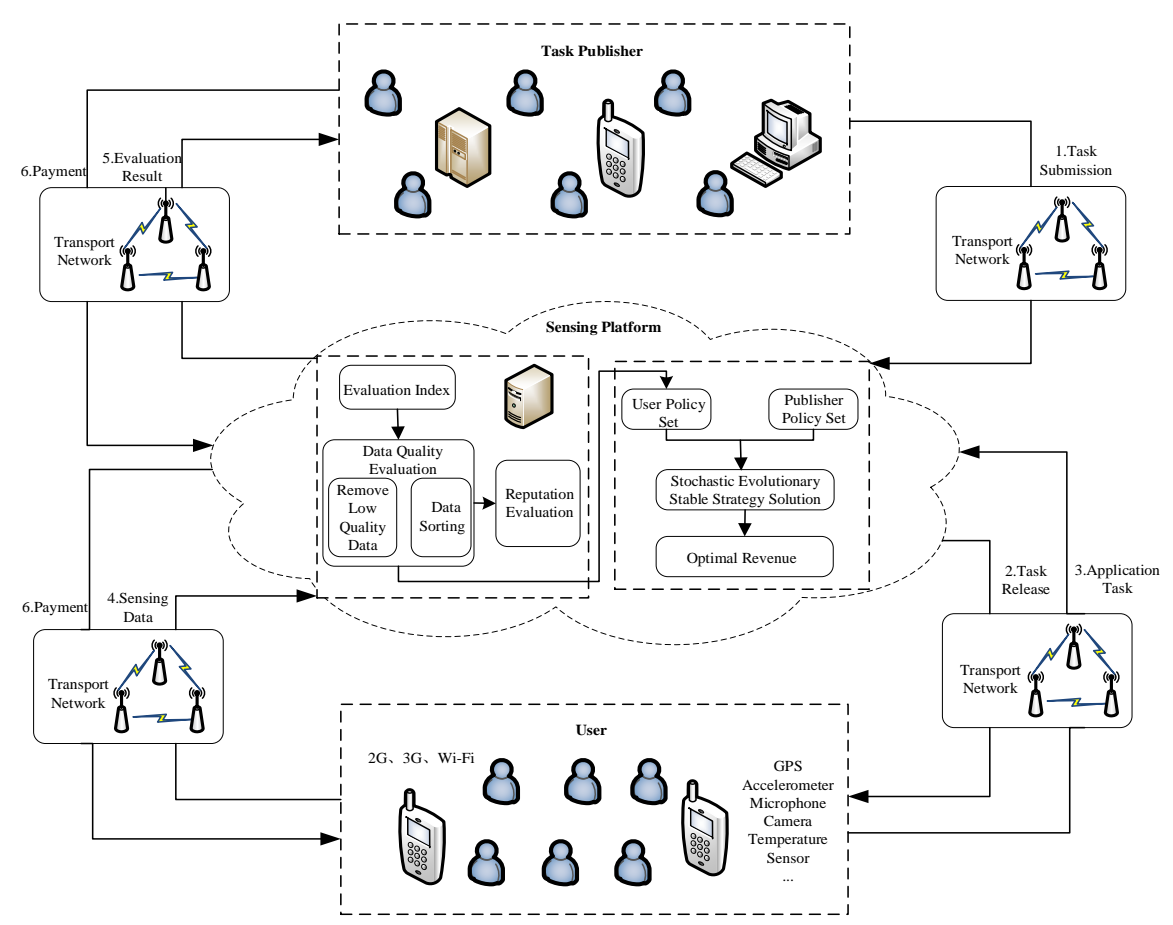

Fig. 2. Task publisher-user stochastic evolutionary game model

The task publisher-user stochastic evolutionary game model is mainly composed of an evaluation module and an optimal payoff strategy selection module. In the evaluation module, firstly, the user credibility is quantified by Logistic function. Secondly, the user's credibility is taken as one of the data quality evaluation indexes. Finally, the data quality evaluation algorithm can remove malicious and low-quality data, and then achieve a reasonable set of 
user data quality strategies. According to the data quality evaluation results, in the optimal payoff strategy selection module, combining the task publisher's incentive strategy set and the user's data quality strategy set. The optimal payoff strategy selection algorithm is used to solve the evolutionary strategy solution and analyze the stability. Finally, the optimal payoff under different initial state and random interference with different intensities is obtained.

Definition 1 . The task publisher-user stochastic evolutionary game model can be expressed as a five-tuple, i.e., $\operatorname{PUSEGM}=(N, V, Q, \Delta, M)$.

(1) $N=\left(N_{u}, N_{p}\right)$ is random evolutionary game participants, where $N_{u}$ denotes the user, and $N_{p}$ denotes the publisher.

(2) $V=(u v, p v)$ is the game strategy space, where $u v=\left\{u v_{1}, u v_{2}, \ldots, u v_{n}\right\}$ denotes users' optional data quality strategy sets, and $p v=\left\{p v_{1}, p v_{2}, \ldots, p v_{m}\right\}$ denotes task publishers' optional incentive strategy sets.

(3) $Q=(q, p)$ is the probability set of the choice strategy of both sides of the game, where $q_{j}$ denotes the probability that users choose $u v_{j}$, and $p_{i}$ denotes the probability that task publishers choose $p v_{i}$.

(4) $\Delta=\left(\delta_{1}, \delta_{2}\right)$ is the set of random interference intensity coefficients, where $\delta_{1}$ denotes the random interference intensity coefficient of the user, and $\delta_{2}$ denotes the random interference intensity coefficient of the publisher.

(5) $M=\left(M_{u}, M_{p}\right)$ is the set of the payoff functions for both sides of the game, where $M_{u}$ denotes the payoff function for the user, and $M_{p}$ denotes the payoff function for the publisher.

\section{Quality and Credibility Evaluation}

Firstly, the user credibility is quantified by logistic function. Secondly, the user's credibility is taken as one of the data quality evaluation indexes. Finally, the data quality of users in the PUSEGM model is improved by the data quality evaluation algorithm (DQEA). And the lowquality data and the malicious data are removed.

\subsection{Quality Evaluation}

In mobile crowdsensing, the payoff of publishers and users is directly proportional to the quality of data submitted by users. Low quality data not only increases the waste of resources of mobile crowdsensing platform, but also reduces the final payoff of task publishers and users. Therefore, data quality evaluation is added to the PUSEGM model to remove the submission of low-quality data immediately. When the user receives the requested task, they use the mobile device to collect data. The data collected contains a lot of information. Because there are multiple sensors in mobile devices, such as time sensors and position sensors. In the evaluation problem of sensing data quality, it is known that there are $m$ users' submitted sensing data, represented as $U=\left\{u_{1}, u_{2}, \ldots, u_{m}\right\}$. There are $n$ data quality evaluation indexes, expressed as $C=\left\{c_{1}, c_{2}, \ldots, c_{n}\right\}$. The decision matrix is $G=\left[g_{i, j}\right]_{m \times n} \cdot g_{i, j}$ is the evaluation value of sensing data $u_{i}$ submitted by users on evaluation index $c_{j}$. The evaluation of data quality is related to the response time of sensors in mobile devices, the accuracy of data collection and the reliability of data transmission. Therefore, according to the classification method proposed by Marjanovic et al. [13], the data quality evaluation indexes are summarized as the following five: 
Distance $\left(c_{1}\right): c_{1}$ is the distance between the user and the task. The closer the user is to the task node, the higher the quality of the data provided by the user. Since the user's mobile devices all have GPS sensors, it is possible to obtain the user's location.

Response time $\left(c_{2}\right): c_{2}$ is the time difference between when the task publisher publishes the task and when the user submits the data.

Data integrity $\left(c_{3}\right): c_{3}$ is the accuracy of data provided by users in different environments. For example, in noise monitoring, data collected in quiet environment is more complete than that collected in noisy environment.

Data reliability $\left(c_{4}\right): c_{4}$ is the accuracy of data provided by users in different states. For example, in noise monitoring, the data provided by the user in the static state is more reliable than that provided in the dynamic state.

Credibility $\left(c_{5}\right): c_{5}$ is a measure of the user's historical contribution behavior. The users with higher credibility are more likely to provide high-quality data than users with lower credibility.

Generally, the evaluation index of data quality is divided into negative correlation index and positive correlation index. The negative correlation index means that the higher the quality of the user's data, the smaller the value of the evaluation index will be. On the contrary, it is a positive correlation index [14]. For the convenience of description, the data quality evaluation indexes are recorded as cost indexes, and benefit indexes, which are expressed by $C_{\text {cost }}$ and $C_{\text {profit }}$ respectively. When the expected range of each index is determined, the malicious data can be judged by the data quality evaluation algorithm, and the data can be sorted according to the error deviation.

The implementation steps of data quality evaluation method are as follows:

Step 1: we calculate the error value $\left(t_{i, j}\right)$ of user data. When $c_{j} \in C_{\text {cost }}$, the calculation $t_{i, j}$ is shown in (1). When $c_{j} \in C_{\text {profit }}$, the calculation $t_{i, j}$ is shown in (2). When the attribute value $g_{i, j}$ is not within the period, $t_{i, j}$ is expressed by the constant $\beta$. In order to ensure that the order of magnitude is the same, $\beta=1+\varepsilon, \varepsilon$ is a positive infinite decimal. When the attribute value $g_{i, j}$ is within the period, the value range of $t_{i, j}$ is $[0,1]$.

$$
\begin{aligned}
& t_{i, j}=\left\{\begin{array}{l}
\beta, g_{i, j}>z_{j}^{\max } \text { or } g_{i, j}<z_{j}^{\min } \\
\frac{g_{i, j}-z_{j}^{\min }}{z_{j}^{\max }-z_{j}^{\min }}, z_{j}^{\min } \leq g_{i, j} \leq z_{j}^{\max }
\end{array}\right. \\
& t_{i, j}=\left\{\begin{array}{l}
\beta, g_{i, j}<z_{j}^{\min } \text { or } g_{i, j}>z_{j}^{\max } \\
\frac{z_{j}^{\max }-g_{i, j}}{z_{j}^{\max }-z_{j}^{\min }}, z_{j}^{\min } \leq g_{i, j} \leq z_{j}^{\max }
\end{array}\right.
\end{aligned}
$$

Where, $z_{j}^{\min }$ and $z_{j}^{\max }$ represent the expected lower limit and expected upper limit of data quality evaluation index respectively. The error value sequence of each user data $u_{i}$ under each data quality evaluation index is denoted as $\left\{t_{i, 1}, t_{i, 2}, \ldots, t_{i, n}\right\}$.

Step 2: we calculate the maximum error value $\left(t_{i}^{\max }\right)$ of user data $u_{i}$ and judge its rationality.

$$
t_{i}^{\max }=\max \left\{t_{i, j}\right\}
$$


When $t_{i}^{\max }=\beta$, it means that the user data is malicious data and this data needs to be removed.

Step 3: we standardize the data quality evaluation indexes and calculate the limit loss value $\left(E_{j}^{*}\right)$ of the data quality evaluation indexes by (4).

$$
\left\{\begin{array}{c}
Z_{j}^{\max }=\frac{z_{j}^{\max }}{z_{j}^{\max }}=1, Z_{j}^{\min }=\frac{z_{j}^{\min }}{z_{j}^{\max }} \\
E_{j}^{*}=\frac{\left(Z_{j}^{\max }-Z_{j}^{\min }\right)^{2}}{\sum_{i=1}^{n}\left(Z_{i}^{\max }-Z_{i}^{\min }\right)^{2}}
\end{array}\right.
$$

Among them, $Z_{j}^{\min }$ and $Z_{j}^{\max }$ respectively represent the expected lower limit and expected upper limit of the standardized processing of data quality evaluation indexes.

Step 4: we calculate the error loss sequence $\left(r_{u_{i}}\right)$ of user data by (5), and sort the data sequence submitted by the user. The tasks published by different task publishers have different concerns about each data quality evaluation index. Therefore, the introduction of weight $\left(\omega_{i}\right)$ can realize different attention and make its data quality more consistent with the needs of task publishers.

$$
r_{u_{i}}=\left\{\omega_{1} * t_{i, 1} * E_{1}^{*}, \omega_{2} * t_{i, 2} * E_{2}^{*}, \ldots, \omega_{j} * t_{i, j} * E_{j}^{*}\right\}
$$

Where, $i=1,2, \ldots, m, j=1,2, \ldots, n, \sum_{j=1}^{n} \omega_{j}=1$. Then, we sort the data by (6). The data error loss sequence $\left(r_{u_{i}}\right)$ is regarded as a coordinate node on the n-dimensional space. The closer the coordinate node is to the origin, the higher the data quality of the user $u_{i}$ will be. We can remove the low quality data and the malicious data by $R_{i, k}$, and finally realize the selection of reasonable user set.

$$
R_{i, k}=\sqrt{\sum_{j=1}^{n}\left(\omega_{j} * t_{i, j} * E_{j}^{*}\right)^{2}}, i=1,2, \ldots, m, k=1,2, \ldots
$$

\subsection{Credibility Evaluation}

After determining the data quality, the user credibility evaluation component is proposed. This component uses the user's historical behavior to evaluate the user's credibility. In mobile crowdsensing, their credibility will increase when users provide high quality data. On the contrary, the user's credibility will be reduced. The famous logistic function is used to update user credibility. Because its characteristic is that the growth speed of left and right sides is slow, the growth speed of middle is fast and the change characteristic of credibility is the same. The credibility $\left(C_{i, k}\left(Q_{i, k}\right)\right)$ is calculated by (7).

$$
C_{i, k}\left(Q_{i, k}\right)=\frac{1}{1+e^{1-Q_{i, k}}}
$$

Where, $k$ is the total number of data submitted by the user. The input parameters of (7) are 
as follows:

$$
\left\{\begin{array}{c}
q_{i, k}=\frac{\frac{1}{R_{i, k} / \lambda+\theta}}{\sum_{j=1}^{m} \frac{1}{R_{j, k} / \lambda+\theta}} \\
Q_{i, k}=\sum_{t=1}^{k} w^{k-t}\left(q_{i, t}-\frac{1}{m}\right)
\end{array}\right.
$$

Where, $\lambda$ is the sum of the distance from the origin of each user's error sequence, $\lambda=\sum_{i=1}^{n} R_{i, k}, \theta$ is a real number. We introduce $\theta$ to ensure that the equation is still meaningful when $R_{i, k}=0$. As shown in 8 , the user's credibility is evaluated by summarizing all the historical quality records in the past. Where, $w^{k-t}(0<w<1)$ is the weight assigned to the data quality in different stages. $q_{i, t}-\frac{1}{m}$ is used to judge whether the data quality of the user for the $t$-th time is higher than the average value. That is, $q_{i, t}-\frac{1}{m}>0$ means that the data quality of users is higher than the average value, and vice versa.

In the actual application scenario, the decline rate and rise rate of user credibility should be different, and should be proportional to the quality of user data. When $q_{i, t} \leq \frac{1}{m}$, we replace the weight with $1-q_{i, t}$. Users with lower data quality have higher aging weights, which leads to the faster the loss of credibility. When $q_{i, t}>\frac{1}{m}$, its aging weight is its quality $q_{i, t}$. Since $q_{i, t}$ is much less than $1-q_{i, t}$, especially when the number of users is large, the decline rate of credibility is always greater than the increase rate of credibility.

$$
Q_{i, k}= \begin{cases}\sum_{t=1}^{k}\left(1-q_{i, t}\right)^{k-t}\left(q_{i, t}-\frac{1}{m}\right) & \text { if } q_{i, t} \leq \frac{1}{m} \\ \sum_{t=1}^{k} q_{i, t}^{k-t}\left(q_{i, t}-\frac{1}{m}\right) & \text { if } q_{i, t}>\frac{1}{m}\end{cases}
$$

Therefore, in order to determine the credibility after the user submits the data for the $k$-th time, (9) is used to calculate $Q_{i, k}$. Then, we use (7) to calculate the credibility, so as to realize the evaluation of the credibility.

\subsection{Data Quality Evaluation Algorithm Description}

In the user's data quality evaluation algorithm, step 1 determines the user's data quality evaluation indexes. The purpose is to quantify the data quality of each user. From step 2 to step 16 use the error elimination decision method to judge whether the user data has malicious data under each index. The low quality and malicious data can be removed and the data quality of users in PUSEGM model can be improved. Step 17 updates the user's credibility. The user's data quality evaluation algorithm can evaluate the user's data quality and eliminate the low quality and the malicious data. It guarantees a reasonable set of user data quality strategy. 


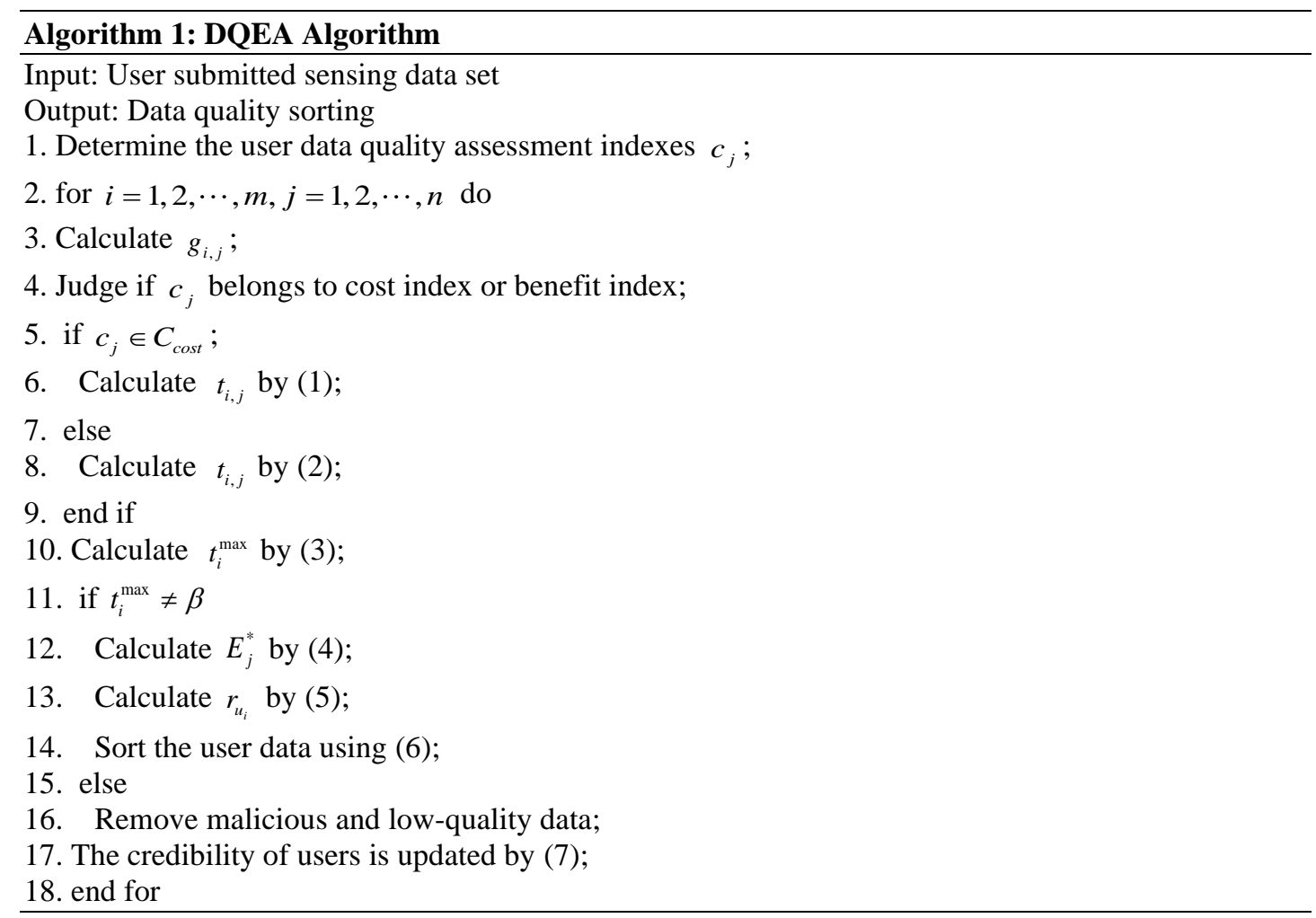

The complexity of the DQEA algorithm is $\mathrm{O}(\mathrm{mn})$. The evaluation indexes in the algorithm can be improved continuously according to the needs of future research, so as to make the data quality evaluation more accurate. Therefore, the data quality evaluation method proposed in this paper has good scalability.

\section{Optimal Payoff Strategy Selection}

Through the data quality assessment method in the previous section, malicious and low-quality data can be removed. Then the reasonable user data quality strategy set is realized. This section combines the task publisher's incentive strategy set and the user's data quality strategy set. The optimal payoff strategy selection algorithm (OPSSA) is used to solve the evolutionary strategy solution and analyze its stability. Finally, the optimal payoff strategy based on evolutionary stability strategy (ESS) is obtained. ESS is an evolutionary stabilization strategy. If most members of a group adopt it, and other strategies cannot produce higher payoff than using this strategy. Then small mutation groups are unlikely to invade this group [15]. This means that in the ESS state, the optimal payoff for task publishers and users can be achieved.

\subsection{Optimal Payoff Strategy Selection Algorithm}

In the PUSEGM model, task publishers and users have multiple incentive strategy sets and multiple data quality strategy sets to choose from. At different stages of the game, the strategy selection of both sides will become a dynamic process. Therefore, the task publisher-user stochastic evolutionary game tree is constructed, as shown in Fig. 3. 


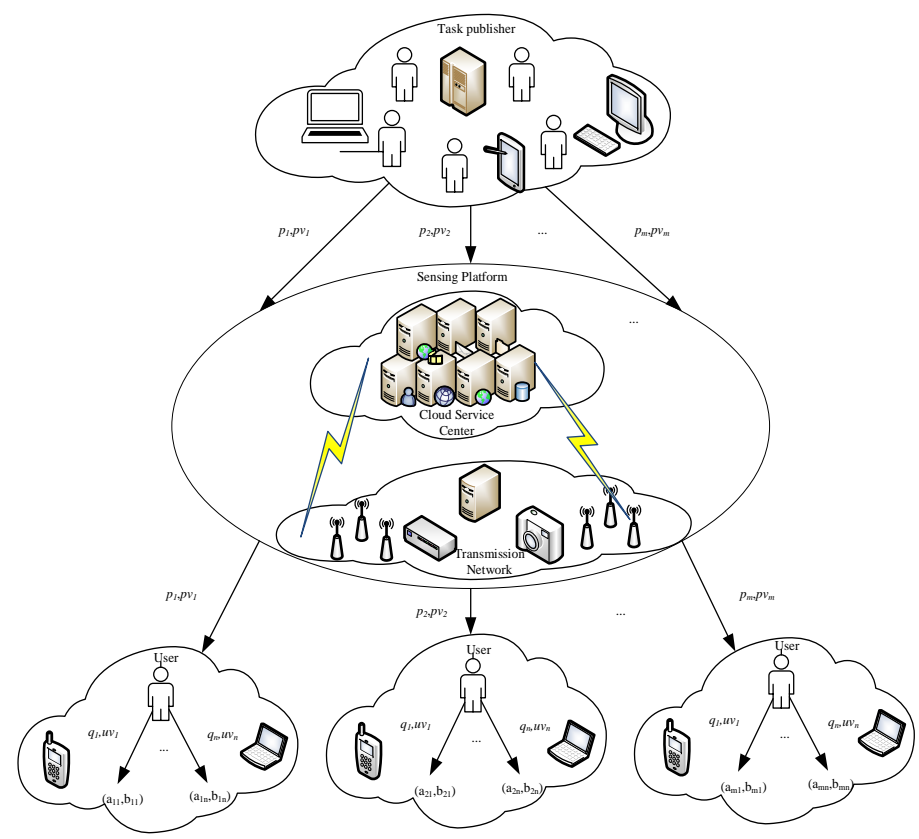

Fig. 3. Task publisher-user stochastic evolutionary game tree

It can be seen from Fig. 3 that when different strategies are adopted, both sides of the game will generate different payoff values. When the task publisher adopts the incentive strategy $i$ and the user adopts the data quality strategy $j, a_{i j}$ is the payoff of the task publisher, and $b_{i j}$ is the payoff of the user. The payoff matrix of both parties is as follows:

$$
\left\{\begin{array}{cccc}
a_{11}, b_{11} & a_{12}, b_{12} & \cdots & a_{1 n}, b_{1 n} \\
a_{21}, b_{21} & a_{22}, b_{22} & \cdots & a_{2 n}, b_{2 n} \\
\vdots & \vdots & \ddots & \vdots \\
a_{m 1}, b_{m 1} & a_{m 2}, b_{m 2} & \cdots & a_{m n}, b_{m n}
\end{array}\right\}
$$

\footnotetext{
Algorithm 2: Optimal Payoff Strategy Selection Algorithm

Input: Task publisher-user random evolutionary game tree Output: Optimal payoff strategy

BEGIN

1. Initialization PUSEGM $=(N, V, Q, \Delta, M)$;

2. $U=\left\{u_{i}, i \geq 1\right\}, P=\left\{p_{i}, i \geq 1\right\} ; / /$ Establish user type space set and task publisher type space set.

3. $p v=\left\{p v_{i}, 1 \leq i \leq n\right\}, u v=\left\{u v_{j}, 1 \leq j \leq m\right\}$;//Establish incentive strategy set of task publisher and user data quality strategy set.

4. $M_{p v_{m}}=q_{1} a_{m 1}+q_{2} a_{m 2}+\cdots+q_{n} a_{m n}=\sum_{i=1}^{n} q_{i} a_{m i} ; / / M_{p v_{m}}$ is the expected payoff of the task publisher's choice of incentive strategy $p v_{i}, q_{i}(1 \leq i \leq n)$ is the probability of user's selection of $u v_{i}$, and $\sum_{i=1}^{n} q_{i}=1, a_{i j}$ is the payoff value of task publisher's selection of $p v_{i}$.

5. $M_{u v_{n}}=p_{1} b_{1 n}+p_{2} b_{2 n}+\cdots+p_{m} b_{m n}=\sum_{j=1}^{m} p_{j} b_{j n} ; / / M_{u v_{n}}$ is the expected payoff of the user's choice of
} 
strategy $u v_{i}, p_{j}(1 \leq j \leq m)$ is the probability of task publisher's selection of $p v_{i}$, and $\sum_{i=1}^{n} p_{i}=1, b_{i j}$ is the payoff value of task publisher's selection of $u v_{i}$.

6. $\overline{M_{p}}=\sum_{i=1}^{m} p_{i} M_{p v_{i}} ; / / \overline{M_{p}}$ is the average payoff of task publisher.

7. $\overline{M_{u}}=\sum_{i=1}^{n} q_{i} M_{u v_{i}} ; / / \overline{M_{u}}$ is the average payoff of user.

8. $\Delta=\left(\delta_{1}, \delta_{2}\right) ; / /$ Establish the set of random interference intensity coefficients for task publishers and users.

9. $d q_{i}(t)=q_{i}(t) *\left(M_{u v_{i}}-\overline{M_{u}}\right) d t+\delta_{1} \sqrt{\left(1-q_{i}(t)\right) q_{i}(t)} d w(t) ; / /$ Users' stochastic differential equation. $w(t)$ belongs to a one-dimensional standard Brown motion, which can well describe how the game evolution process of task publisher and user is affected by random interference factors. $w(t)$ follows the normal distribution $N(0, t) . d w(t)$ is random interference. When $t>0$ and $h>0$, $\Delta w(t)=w(t+h)-w(t)$ follows the normal distribution $N(0, \sqrt{h})$.

10. $d p_{i}(t)=p_{i}(t) *\left(M_{p v_{i}}-\overline{M_{p}}\right) d t+\delta_{2} \sqrt{\left(1-p_{i}(t)\right) p_{i}(t)} d w(t) ; / /$ Task publishers' stochastic differential equation.

11. Combining the random taylor expansion [16] and itó random formula [17], taylor expansion of stochastic differential equations is carried out;

12. Milstein method [18] is used to solve the stochastic differential equation;

13. Output optimal payoff strategy; END

The calculation process of OPSSA algorithm is analyzed. The time complexity from step 1 to step 7 is $\mathrm{O}(\mathrm{m}+\mathrm{n})$. The time complexity from step 8 to step 12 is $\mathrm{O}\left((\mathrm{m}+\mathrm{n})^{2}\right)$. In summary, the time complexity of solving the optimal payoff strategy is $\mathrm{O}\left((\mathrm{m}+\mathrm{n})^{2}\right)$. The consumption of storage space is mainly focused on the storage of strategic payoff and the equilibrium solution of the intermediate value, and its space complexity is $\mathrm{O}(\mathrm{mn})$. Through the analysis of game results, OPSSA algorithm obtains the payoff and evolution stable state of each strategy, and realizes the prediction of strategy selection. From the perspective of optimal payoff, this is a model that guides the choice of strategy for task publishers and users. Because the number of strategies can be increased, this method is universal.

\subsection{Example Description}

The PUSEGM model is illustrated by a simple example. Assuming that the task publisher is $p$ and the user is $u$. In order to simplify the calculation complexity, the user's data quality strategy only uses two types of $\left\{u v_{l}, u v_{h}\right\}$, and the task publisher's incentive strategy also uses two types of $\left\{p v_{l}, p v_{h}\right\}$. Because the number of people choosing different strategies will change over time, $q(t)$ is used to represent the probability of user selecting strategy $u v_{l} .1-q(t)$ is used to represent the probability of user selecting strategy $u v_{h} \cdot p(t)$ is used to represent the probability of task publisher selecting strategy $p v_{l} .1-p(t)$ is used to represent the probability of task publisher selecting strategy $p v_{h}$. 


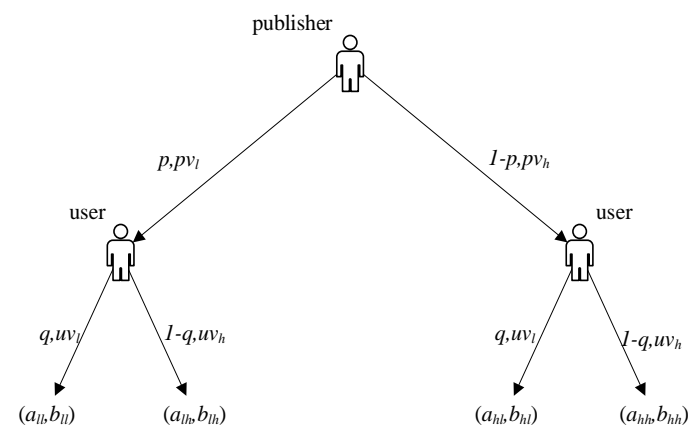

Fig. 4. Simple task publisher-user random evolutionary game tree

In the example shown in Fig. 4, in the PUSEGM model, the strategic payoffs of both parties should meet the following conditions: $a_{h h}>a_{l h}>a_{l l}>a_{h l}$ and $b_{h h}>b_{h l}>b_{l l}>b_{l h}$. According to step 9 of the OPSSA algorithm, the user's stochastic differential equation in the model can be obtained:

$$
\begin{aligned}
d q(t) & =q(t)\left[p(t)\left(b_{l l}-b_{h l}-b_{l h}+b_{h h}\right)\right. \\
& \left.+\left(b_{l h}-b_{l l}\right)\right] d t+\delta_{1} \sqrt{(1-q(t)) q(t)} d w(t)
\end{aligned}
$$

Similarly, according to step 10 of the OPSSA algorithm, the task publisher stochastic differential equation in the model can be obtained:

$$
\begin{aligned}
d p(t) & =p(t)\left[q(t)\left(a_{l l}-a_{h l}-a_{l h}+a_{h h}\right)\right. \\
& \left.+\left(a_{h l}-a_{l l}\right)\right] d t+\delta_{2} \sqrt{(1-p(t)) p(t)} d w(t)
\end{aligned}
$$

Evolutionary equilibrium solution: since the stochastic taylor expansion is the basis for the numerical solution of stochastic differential equation. The milstein method is based on the stochastic taylor expansion to solve the stochastic differential equation. Therefore, we first need to combine stochastic taylor expansion [16] and itó stochastic formula [17] to perform taylor expansion on stochastic differential equation. Then, the milstein method [18] is used to numerically solve the stochastic differential equation, and finally the evolutionary equilibrium solution of the stochastic differential equation is obtained. For the following itó stochastic differential equation [19]:

$$
d x(t)=f(t, x(t)) d t+g(t, x(t)) d w(t)
$$

Where, $t \in\left[t_{0}, T\right], x\left(t_{0}\right)=x_{0}, x_{0} \in R, w(t)$ follows the normal distribution $N(0, t), d(w(t))$ follows the normal distribution $N(0, \Delta t)$. Let $h=\left(T-t_{0}\right) / N$ and $t_{n}=t_{0}+n h$, the stochastic taylor expansion of equation (12) can be obtained as follows:

$$
\begin{aligned}
x\left(t_{n+1}\right) & =x\left(t_{n}\right)+M_{0} f\left(x\left(t_{n}\right)\right) d t+M_{1} g\left(x\left(t_{n}\right)\right) \\
& +M_{11} N^{1} g\left(x\left(t_{n}\right)\right)+M_{00} N^{0} f\left(x\left(t_{n}\right)\right)+R
\end{aligned}
$$

Where, $R$ represents the remainder of the expansion and satisfies the following conditions:

$$
N^{0}=f(x) \frac{\partial}{\partial x}+\frac{1}{2} g^{2}(x) \frac{\partial^{2}}{\partial x^{2}} ; N^{1}=g(x) \frac{\partial}{\partial x} ; M_{0}=h ; M_{1}=\Delta w_{n} ; M_{00}=\frac{1}{2} h^{2} ; M_{11}=\frac{1}{2}\left[(\Delta w)^{2}-h\right]
$$


Based on this, the milstein method [18] is used to numerically solve itó stochastic differential equation, and the following can be obtained:

$$
\begin{array}{r}
x\left(t_{n+1}\right)=x\left(t_{n}\right)+h f\left(x\left(t_{n}\right)\right)+\Delta w_{n} g\left(x\left(t_{n}\right)\right) \\
+\frac{1}{2}\left[\left(\Delta w_{n}\right)^{2}-h\right] g\left(x\left(t_{n}\right)\right) g^{\prime}\left(x\left(t_{n}\right)\right)
\end{array}
$$

According to the above solution to the itó stochastic differential equation, the differential (10) and (11) can be solved numerically, and the corresponding task publisher-user stochastic evolution equilibrium solution can be obtained.

For the existence of the above equilibrium solution, according to the stability discrimination theorem of stochastic differential equation [20], the stability analysis is carried out for the strategy selection of the PUSEGM model.

Proposition 1. For (10), let $p=1, \gamma=1, q(t) \in[0,1], V(t, q(t))=q(t), c_{1}=c_{2}=1$, then $L V(t, q(t))=f(t, q(t))$. Then satisfy:

(1) When $\left\{\begin{array}{l}p(t) \leq \frac{b_{l l}-b_{l h}-1}{b_{l l}-b_{h l}-b_{l h}+b_{h h}} \\ b_{l l}-b_{l h} \geq 1\end{array}\right.$ the expected moment of the zero solution of equation (10) is exponentially stable;

(2) When $\left\{\begin{array}{l}p(t) \geq \frac{b_{l l}-b_{l h}+1}{b_{l l}-b_{h l}-b_{l h}+b_{h h}} \text {, the expected moment of the zero solution of equation } \\ 1-b_{h h}+b_{h l} \leq 0\end{array}\right.$ (10) is exponentially instable.

Proof: (1) For (10), let $p=1, \gamma=1, c_{1}=c_{2}=1, q(t) \in[0,1], V(t, q(t))=q(t)$, then $L V(t, q(t))=f(t, q(t))=q(t)\left[p(t)\left(b_{l l}-b_{h l}-b_{l h}+b_{h h}\right)+\left(b_{l h}-b_{l l}\right)\right]$, to make (10) satisfy the exponential stability of the expected moment of zero solution, we need to meet the following requirements: $L V(t, q(t)) \leq-\gamma V(t, q(t))$, By $q(t) \in[0,1]$, we can get:

$$
p(t)\left(b_{l l}-b_{h l}-b_{l h}+b_{h h}\right)-\left(b_{l l}-b_{l h}-1\right) \leq 0
$$

In the PUSEGM model, the strategic payoff of both parties meet the following conditions: $a_{h h}>a_{l h}>a_{l l}>a_{h l}$ and $b_{h h}>b_{h l}>b_{l l}>b_{l h}$, we can see that $b_{l l}-b_{h l}-b_{l h}+b_{h h}>0$, we can get:

Proof completed.

$$
p(t) \leq \frac{b_{l l}-b_{l h}-1}{b_{l l}-b_{h l}-b_{l h}+b_{h h}} \text { and } b_{l l}-b_{l h} \geq 1
$$

(2) To make (10) satisfy the exponential instability of the expected moment of zero solution, we need to meet the following requirements: $L V(t, q(t)) \geq \gamma V(t, q(t))$, By $q(t) \in[0,1]$, we can get:

$$
p(t)\left(b_{l l}-b_{h l}-b_{l h}+b_{h h}\right)-\left(b_{l l}-b_{l h}+1\right) \geq 0
$$

By $b_{l l}-b_{h l}-b_{l h}+b_{h h}>0$, we can get: 


$$
p(t) \geq \frac{b_{l l}-b_{l h}+1}{b_{l l}-b_{h l}-b_{l h}+b_{h h}} \text { and } 1-b_{h h}+b_{h l} \leq 0
$$

Proof completed.

Proposition 2: For (11), let $q=1, \gamma=1, V(t, q(t))=q(t), p(t) \in[0,1], c_{1}=c_{2}=1$, then $L V(t, p(t))=f(t, p(t))$. Then satisfy:

(1) When $\left\{\begin{array}{l}q(t) \leq \frac{a_{l l}-a_{h l}-1}{a_{l l}-a_{h l}-a_{l h}+a_{h h}} \\ a_{l l}-a_{h l} \geq 1\end{array}\right.$ the expected moment of the zero solution of equation (11) is exponentially stable;

(2) When $\left\{\begin{array}{l}q(t) \geq \frac{a_{l l}-a_{h l}+1}{a_{l l}-a_{h l}-a_{l h}+a_{h h}}, \text { the expected moment of the zero solution of equation (11) } \\ 1-a_{h h}+a_{l h} \leq 0\end{array}\right.$ is exponentially instable.

Proof: The method of proof is consistent with proposition 1.

From proposition 1 and proposition 2, when $\left\{\begin{array}{l}p(t) \leq \frac{b_{l l}-b_{l h}-1}{b_{l l}-b_{h l}-b_{l h}+b_{h h}} \\ b_{l l}-b_{l h} \geq 1\end{array}\right.$ and $\left\{\begin{array}{l}p(t) \geq \frac{b_{l l}-b_{l h}+1}{b_{l l}-b_{h l}-b_{l h}+b_{h h}} \\ 1-b_{h h}+b_{h l} \leq 0\end{array}\right.$, PUSEGM model has a unique evolutionary stability strategy $\operatorname{ESS}(0,0)$. Task publishers choose low incentive strategy and users provide low-quality data. And When

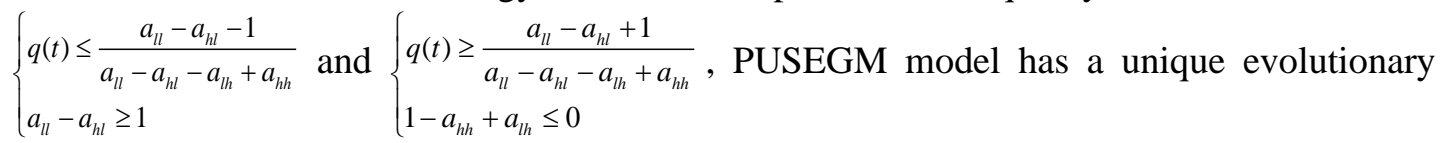
stability strategy $\operatorname{ESS}(1,1)$. That is, task publishers choose high incentive strategies and users provide high-quality data. This is consistent with the continuous evolution of actual task publishers and users.

\section{Simulation Analysis}

\subsection{Simulation Environment}

CRAWDAD, the data set used in the experiment, is a public community resource of wireless network data of Dartmouth College. The dataset uses open-source middleware Nsense to collect continuous tracking data based on four sensors, i.e. Bluetooth, Wi-Fi, Microphone and Accelerometer [21]. According to the classification method of reference [13], the data quality evaluation indexes are divided into distance, response time, data integrity, data reliability and credibility. The results after index quantification are shown in Table 1.

Table 1. Index quantification

\begin{tabular}{|c|c|}
\hline Index & Quantification Range \\
\hline Distance $\left(c_{1}\right) / \mathrm{m}$ & {$[10,90]$} \\
\hline Response time $\left(c_{2}\right) / \mathrm{min}$ & {$[0,5000]$} \\
\hline \multirow{2}{*}{ Data integrity $\left(c_{3}\right)$} & Quiet $[0.75,0.9]$ \\
\cline { 2 - 2 } & Normal $[0.6,0.75)$ \\
\hline
\end{tabular}




\begin{tabular}{|c|c|}
\hline \multirow{2}{*}{ Data reliability $\left(c_{4}\right)$} & Alert[0.45,0.6) \\
\cline { 2 - 2 } & Noisy[0.3,0.45] \\
\hline \multirow{2}{*}{ Credibility $\left(c_{5}\right)$} & Stationary[0.7,0.9] \\
\cline { 2 - 2 } & Walking $[0.5,0.7)$ \\
\cline { 2 - 2 } & Running $[0.3,0.5]$ \\
\hline
\end{tabular}

\subsection{Analysis of Data Quality Evaluation Results}

In MCS, the high-precision data quality evaluation can select a reasonable set of users to complete the task, so that task publishers and users can get the best payoff. This method is compared with BP detection method and SVM detection method, so as to compare the accuracy of anomaly data detection $\left(A c c_{a}\right)$ and data classification $\left(A c c_{b}\right) . A c c_{a}$ and $A c c_{b}$ are calculated by (15).

$$
\left\{\begin{array}{l}
\operatorname{Acc}_{a}=\frac{\operatorname{sum}_{a b}}{\operatorname{sum}_{a}} \\
\operatorname{Acc}_{b}=\frac{\operatorname{sum}_{d}}{\operatorname{sum}_{b}}
\end{array}\right.
$$

Where, sum ad is the number of real abnormal data in the abnormal data, sum is the number of abnormal data, sum $_{d}$ is the number of data judged correctly, and $\operatorname{sum}_{b}$ is the number of data. The comparison between $A c c_{a}$ and $A c c_{b}$ is shown in Fig. 5.

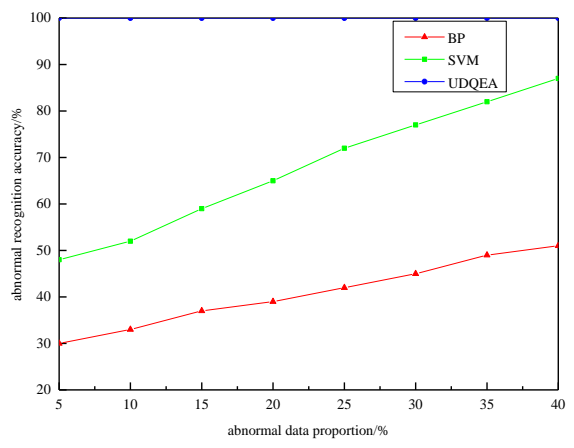

(a)

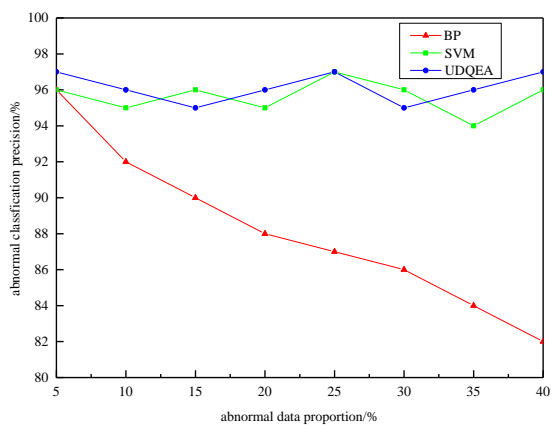

(b)

Fig. 5. $A c c_{a}$ and $A c c_{b}$; (a) Outlier data detection accuracy (b) Data classification accuracy

It can be seen from Fig. 5(a) that with the increase of abnormal data, the detection accuracy of BP detection method and SVM detection method is improved. This is because with the increase of training samples, the detection accuracy of these two methods will be improved. At the same time, it can be seen that the detection accuracy of abnormal data of DQEA method is 1. Compared with BP detection method and SVM detection method, the accuracy of anomaly data detection of DQEA method is improved by $8.1 \%$ and $0.5 \%$ respectively. When there is data deviation, the DQEA method is very sensitive to the data, so as to identify it as abnormal data. The advantage of DQEA method is that it requires less training samples and high accuracy in identifying abnormal data. It can be seen from Fig. 5(b) that with the increase 
of abnormal data, the classification accuracy of DQEA method for data is above $96 \%$. Compared with BP detection method and SVM detection method, the accuracy of data classification of DQEA method is improved by $59.2 \%$ and $32.2 \%$ respectively. This shows that the DQEA method has the advantages of high accuracy and anti-interference in data classification.

\subsection{Analysis of Optimal Payoff Strategy Results}

Aiming at the proposed PUSEGM model and solution analysis process, the system dynamics is used for simulation verification. This paper analyzes the problem of selecting the optimal payoff strategy for task publishers and users in the PUSEGM model. By changing the random interference intensity coefficient of task publisher and user, we can observe its influence on game evolution of both sides. The milstein method was used for numerical simulation. And the random interference intensity coefficients of user and publisher are calculated as $\delta_{1}=0.5, \delta_{1}=2, \delta_{1}=5$ and $\delta_{2}=0.5, \delta_{2}=2, \delta_{2}=5$, which was used to analyze the evolution law of task publisher and user strategies under different random interference. The selection of optimal payoff strategy for task publisher and user under different initial state is given. Simulation can observe the evolution trend of task publisher and user selection strategies under different random interference and different initial state. The final evolutionary stable state is obtained and the optimal payoff strategy for task publisher and user is selected.

In the simulation test, according to the quantitative method proposed in reference [22], two groups of payoff parameters are given. The value of the first set of payoff parameters are $a_{h h}=20, a_{h l}=16, a_{l l}=12, a_{l h}=2, \quad b_{h h}=15, \quad b_{h l}=12, b_{l l}=8, b_{l h}=0, \quad$ calculated $\frac{a_{l l}-a_{h l}-1}{a_{l l}-a_{h l}-a_{l h}+a_{h h}}=\frac{9}{14}$, $a_{l l}-a_{h l} \geq 1$ and $\frac{b_{l l}-b_{l h}-1}{b_{l l}-b_{h l}-b_{l h}+b_{h h}}=\frac{7}{11}, 1-b_{h h}+b_{h l} \leq 0$. And the value of the second set of payoff parameters are $a_{h h}=20, a_{h l}=10, a_{l l}=5, a_{l h}=1, b_{h h}=15, b_{h l}=12, b_{l l}=8, b_{l h}=0 \quad, \quad$ calculated $\frac{a_{l l}-a_{h l}+1}{a_{l l}-a_{h l}-a_{l h}+a_{h h}}=\frac{5}{14}, 1-a_{h h}+a_{l h} \leq 0$ and $\frac{b_{l l}-b_{l h}+1}{b_{l l}-b_{h l}-b_{l h}+b_{h h}}=\frac{4}{11}, 1-b_{h h}+b_{h l} \leq 0$.

\subsubsection{Optimal Payoff Strategy under Different Initial State}

Assuming that the random interference is 0.5 , the evolution trend of strategy selection under different initial state is observed by changing the initial state. In the following evolution trend chart, the abscissa time is time, and the ordinate proportion represents the probability of selecting strategy. It can be seen from Fig. 6 that given different initial state of strategy selection, the strategy selection will eventually reach a certain stable state after evolution. In different initial states, the final stable equilibrium solution of the system is different. It can be seen from Fig. 6(a) and Fig. 6(c) that both sides of the game eventually tend to $\operatorname{ESS}(0,0)$. Thus, the system reaches equilibrium state. Task publishers are more likely to adopt the lowincentive strategy and users are more likely to adopt the low-data quality strategy. So that task publishers and users can get the best payoff at the same time. It can be seen from Fig. 6(b) and Fig. 6(d) that both sides of the game eventually tend to $\operatorname{ESS}(1,1)$.Thus, the system reaches equilibrium state. Task publishers are more likely to adopt the high-incentive strategy and users are more likely to adopt the high-data quality strategy. So that task publishers and users can get the best payoff at the same time. 


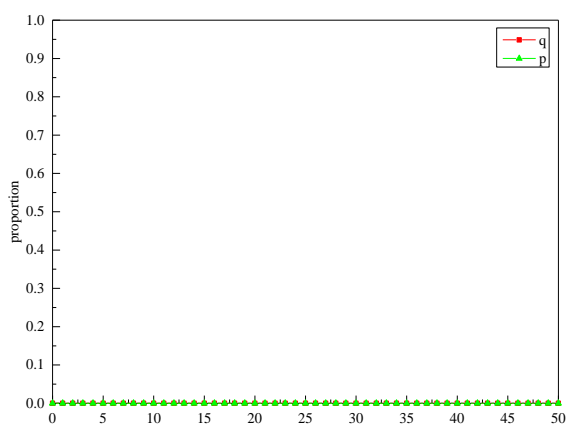

(a)

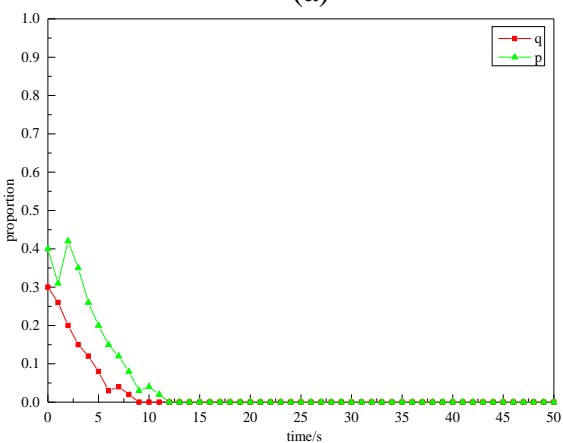

(c)

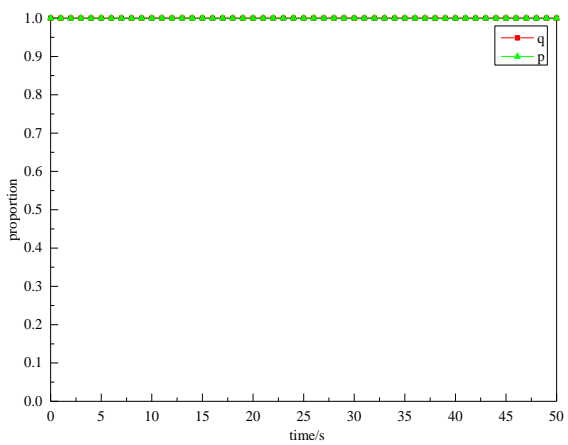

(b)

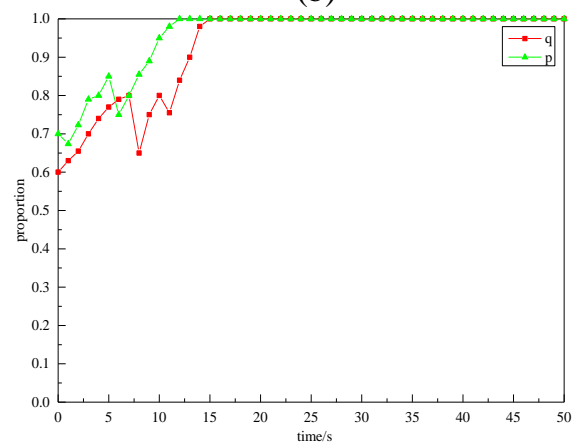

(d)

Fig. 6. When $\delta_{1}=0.5$, the evolution curve under different initial states; (a) The initial state is $p(0)=0, q(0)=0$ (b) The initial state is $p(0)=1, q(0)=1$ (c) The initial state is $p(0)=0.4, q(0)=0.3(\mathrm{~d})$ The initial state is $p(0)=0.7, q(0)=0.6$

\subsubsection{Optimal Payoff Strategy under Different Random Interference}

Assuming that the initial state $p(0)=0.5, q(0)=0.5$ is selected as the strategy. By changing the random interference intensity coefficient, the influence of random interference intensity on the evolution of the game of both sides is observed. In the case of the first set of payoffs, $p(0)$ satisfies the zero moment exponential stability condition of (10), and the user is finally stable at the state of $q(t)=0$. That is, the user chooses the low data quality strategy. At the same time, $q(0)$ satisfies the zero moment exponential stability condition of (11), and the task publisher is finally stable at the state of $p(t)=0$. That is, the task publisher chooses the low incentive strategy. Through system simulation and evolution, the experimental results are shown in Fig. 7(a) and Fig. 7(b). In the case of the second set of payoffs, $p(0)$ satisfies the zero moment exponential instability condition of (10), and the user is finally stable at the state of $q(t)=1$. That is, the user chooses the high data quality strategy. At the same time, $q(0)$ satisfies the zero moment exponential instability condition of (11), and the task publisher is finally stable at the state of $p(t)=1$. That is, the task publisher chooses the high incentive strategy. Through system simulation and evolution, the experimental results are shown in Fig. 7(c) and Fig. 7(d).

It can be seen from Fig. 7 that different random interference intensity has different influence on the evolution rate of game system between task publisher and user. It can be seen from Fig. 7(a) and Fig. 7(b) that with the decrease of $\delta_{1}$ and $\delta_{2}$, the less time it takes for the task publisher and user selection strategy to reach a stable state. It shows that the smaller $\delta_{1}$ 
and $\delta_{2}$, the two sides of the game will eventually tend to $\operatorname{ESS}(0,0)$. So that the system reaches an equilibrium state, task publishers are more inclined to adopt $p v_{l}$ and users are more inclined to adopt $u v_{l}$. The task publisher and user can obtain the optimal payoff at the same time. It can be seen from Fig. 7(c) and Fig. 7(d) that the time required for the evolution of task publisher and user selection strategies to reach a stable state is less with the increase of $\delta_{1}$ and $\delta_{2}$. It shows that the larger $\delta_{1}$ and $\delta_{2}$, the two sides of the game will eventually tend to ESS $(1,1)$. So that the system reaches a balanced state, task publishers tend to adopt $p v_{h}$ and users tend to adopt $u v_{h}$. The task publisher and user can obtain the optimal payoff at the same time. It can be seen from the above simulation results that when there are random interference factors, the PUSEGM model can prevent the interference from destroying the stability by changing the strategies of task publishers and users. It shows that the PUSEGM model conforms to the evolution law of mobile crowdsensing strategy in reality. Under different states, when the system reaches the evolutionarily stable state $\operatorname{ESS}(0,0)$ and $\operatorname{ESS}(1,1)$, the task publisher and the user can obtain the optimal payoff at the same time.

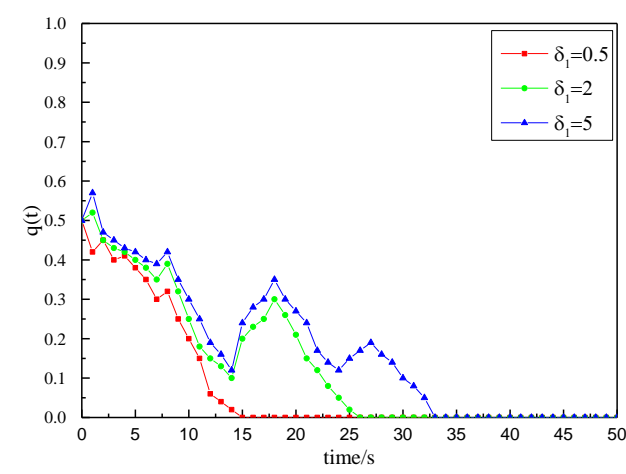

(a)

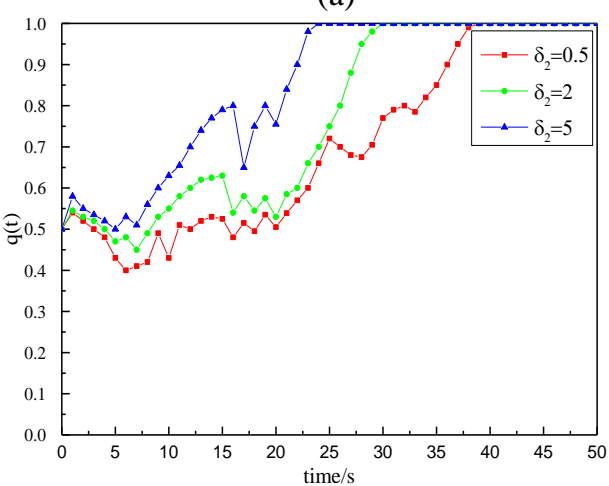

(c)

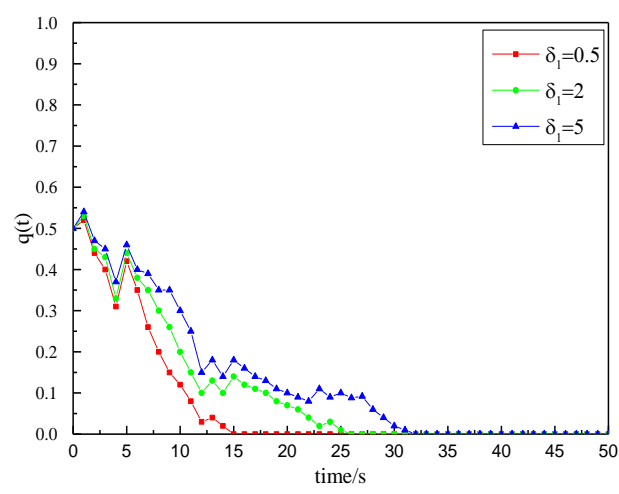

(b)

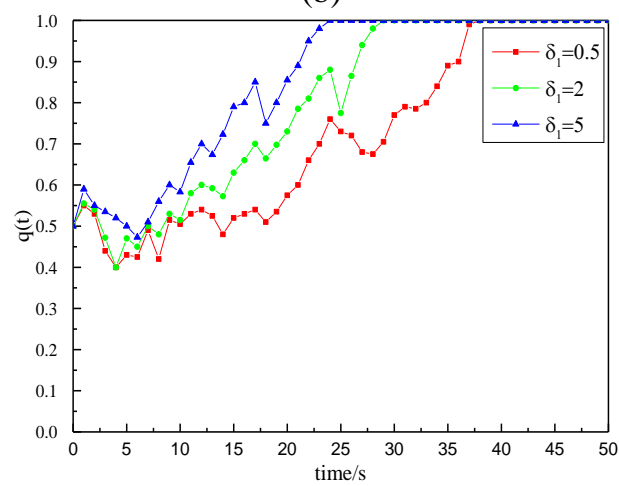

(d)

Fig. 7. When $p(0)=0.5, q(0)=0.5$, the evolution curve under different random interference; (a)

User's zero solution stabilization strategy (b) Task publisher's zero solution stabilization strategy (c) User's zero solution instabilization strategy (d) Task publisher's zero solution instabilization strategy

\subsubsection{Payoff Comparison}

Use the stochastic game model and the PUSEGM model to conduct a comparative simulation. The average payoff of task publishers and users is counted every 10 minutes, and the statistics are made 10 times. The result is shown in Fig. 8. It can be seen from Fig. 8 that the payoff of 
task publisher and user in PUSEGM model is higher than that of task publisher and user in stochastic game model. The reason is that the stochastic game model does not consider people's bounded rationality and learning ability, the payoff is low. The PUSEGM model considers the bounded rationality and learning ability of people. After evolution, the model obtains the evolutionary stability strategy, and finally obtains the optimal payoff strategy based on the evolutionary stability strategy. Evolutionary stabilization strategy means that if most members of a group adopt it, and other strategies cannot produce higher payoff than using this strategy. Then small mutation groups are unlikely to invade this group. This means that in the evolutionary stability strategy state, the optimal payoff for task publishers and users can be achieved. Therefore, the payoff of the PUSEGM model is higher than that of the stochastic game model.

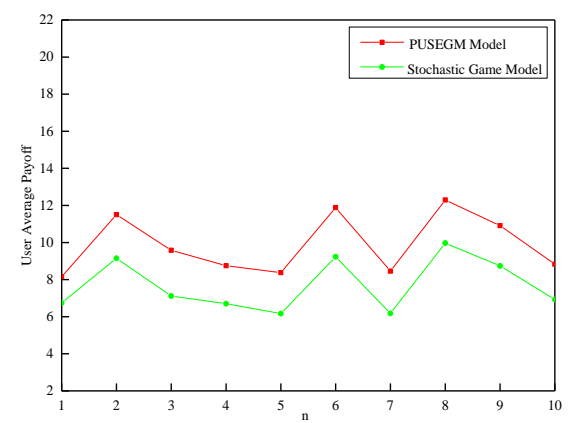

(a)

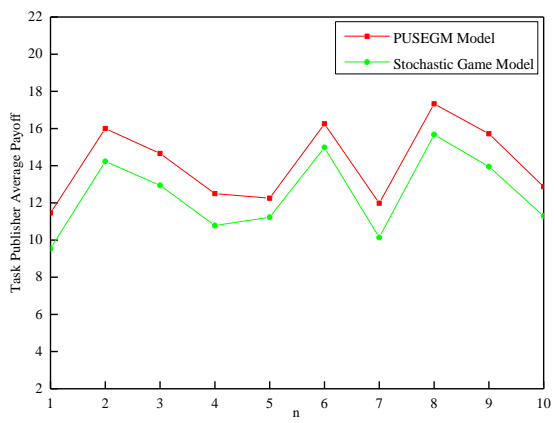

(b)

Fig. 8. Payoff curve of task publisher and user; (a) Comparison of average payoff of users (b)Comparison of average payoff of task publishers

In order to verify the impact of data quality evaluation methods on the payoff of task publishers and users. The average payoff of task publishers and users in the first 30 minutes with and without data quality evaluation were respectively counted for 10 times. The results are shown in Fig. 9.

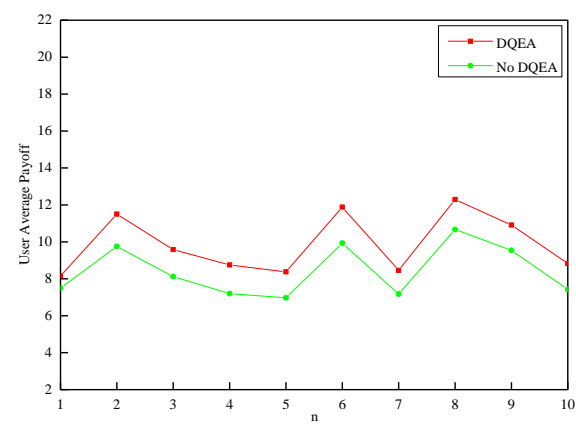

(a)

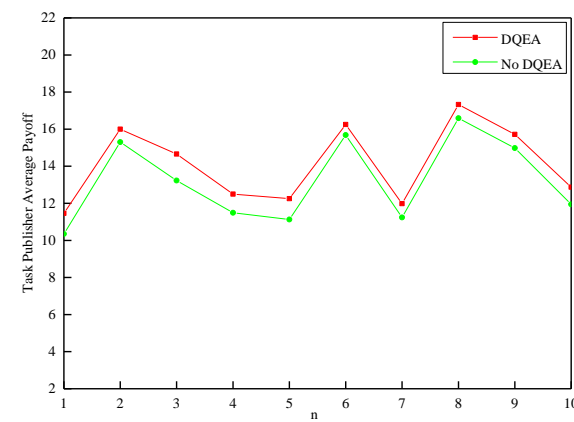

(b)

Fig. 9. Payoff curve of task publisher and user; (a) Comparison of average payoff of users (b)Comparison of average payoff of task publishers

Fig. 9 proves that the game model with data quality evaluation has higher payoff than the game model without data quality evaluation. Because the data quality evaluation removes lowquality data and malicious data, the payoff of task publishers and legitimate users are increased. 


\section{Conclusion}

Aiming at the selection of task payoff strategy in the mobile crowdsensing, we propose a task publisher-user stochastic evolutionary game model. The main works are as follows: (1)The data quality evaluation algorithm is proposed, which can remove the low-quality data. According to the task requirements, the data is selected and the reasonable user set is guaranteed, so that the task publisher and the user can obtain the optimal payoff. (2) The optimal payoff strategy selection algorithm is proposed. The optimal payoff strategy under different intensity of random interference and different initial state is obtained by solving the stability strategy and analyzing the stability of the model. According to the requirements of different tasks, this model can establish different data quality evaluation indexes. At the same time, the influence of random factors is considered in the process of payoff strategy selection. Therefore, the proposed model has good scalability and practical significance. The next step is to make a more comprehensive explanation and analysis of the random interference factors in the model.

\section{Acknowledgement}

This present research work was supported by the National Natural Science Foundation of China (No.61202458, 61403109), the Natural Science Foundation of Heilongjiang Province of China (No.LH2020F034) and the Harbin Science and Technology Innovation Research Funds (No.2016RAQXJ036).

\section{References}

[1] X. L. Zhang, Y. Zhen, W. Sun, W. Liu, S. Tang, K. Xing, and X. Mao, "Incentives for Mobile Crowd Sensing: A Survey," IEEE Communications Surveys \& Tutorials, vol. 18, no. 1, pp. 54-67, 2016. Article (CrossRef Link)

[2] O. Alvear, C. Calafate, J. C. Cano, and P. Manzoni, "Crowdsensing in Smart Cities: Overview, Platforms, and Environment Sensing Issues," Sensors, vol. 18, no. 2, 2018. Article (CrossRef Link)

[3] Y. C. Tung and K. G. Shin, "Echo Tag: accurate infrastructure-free indoor location tagging with smartphones," in Pro. of the $21^{\text {st }}$ Annual International Conference on Mobile Computing and Networking, pp. 525-536, 2015. Article (CrossRef Link)

[4] X. Wang, J. B. Zhang, X. H. Tian, Y. Guan, and X. Wang, "Crowdsensing-Based Consensus Incident Report for Road Traffic Acquisition," IEEE Transactions on Intelligent Transportation Systems, vol. 19, no. 8, pp. 2536-2547, 2018. Article (CrossRef Link)

[5] M. Pouryazdan, C. Fiandrino, B. Kantarci, T. Soyata, D. Kliazovich, and P. Bouvry, "Intelligent Gaming for Mobile Crowd-Sensing Participants to Acquire Trustworthy Big Data in the Internet of Things," IEEE Access, vol. 5, no. 99, pp. 22209-22223, 2017. Article (CrossRef Link)

[6] J. T. Nie, J. Luo, Z. H. Xiong, D. Niyato, and P. Wang, "A Stackelberg Game Approach Toward Socially-Aware Incentive Mechanisms for Mobile Crowdsensing," IEEE Transactions on Wireless Communications, vol. 18, no. 1, pp. 724-738, 2019. Article (CrossRef Link)

[7] X. Liu, K. Ota, A. Liu, and Z. Chen, "An incentive game based evolutionary model for crowd sensing networks," Peer-to-Peer Networking and Applications, vol. 9, no. 4, pp. 692-711, 2016. Article (CrossRef Link)

[8] R. Na, L. Gao, H. Zhu, W. Jia, X. Li, and Q. Hu, "Toward Optimal DoS-Resistant Authentication in Crowdsensing Networks via Evolutionary Game," in Proc. of the IEEE $36^{\text {th }}$ International Conference on Distributed Computing Systems, pp. 364-373, June 2016. Article (CrossRef Link) 
[9] G. Yang, S. B. He, Z. G. Shi, and J. Chen, "Promoting Cooperation by the Social Incentive Mechanism in Mobile Crowdsensing," IEEE Communications Magazine, vol. 55, no. 3, pp. 8692, 2017. Article (CrossRef Link)

[10] D. Peng, F. Wu, and G. H. Chen, "Data Quality Guided Incentive Mechanism Design for Crowdsensing," IEEE Transactions on Mobile Computing, vol. 17, no. 2, pp. 307-319, 2018. Article (CrossRef Link)

[11] S. Yang, F. Wu, S. J. Tang, X. Gao, B. Yang, and G. Chen, "On Designing Data Quality-Aware Truth Estimation and Surplus Sharing Method for Mobile Crowdsensing," IEEE Journal on Selected Areas in Communications, vol. 35, no. 4, pp. 832-847, 2017. Article (CrossRef Link)

[12] E. Wang, Y. J. Yang, and K. H. Lou, "User selection utilizing data properties in mobile crowdsensing," Information Sciences, vol. 490, pp. 210-226, 2019. Article (CrossRef Link)

[13] M. Marjanovic, L. SkorinKapov, K. Pripuzic, A. Antonic, and I. P. Zarko, "Energy-aware and quality-driven sensor management for green mobile crowd sensing," Journal of Network and Computer Applications, vol. 59, pp. 95-108, 2016. Article (CrossRef Link)

[14] Y. M. Lin, H. Z. Wang, S. Zhang, J. Li, and H. Gao, "Efficient quality-driven source selection from massive data sources," Journal of Systems and Software, vol. 118, pp. 221-233, 2016. Article (CrossRef Link)

[15] T. Behrouz, J. Hassan, and J. S. Shamma, "Stochastic Evolutionary Dynamics: A Graphical Reformulation of Evolutionarily Stable Strategy (ESS) Analysis," IEEE Control Systems Letters, vol. 3, no. 1, pp. 55-60, 2019. Article (CrossRef Link)

[16] G. I. Bischi, U. Merlone, and E. Pruscini, "Evolutionary dynamics in club goods binary games," Journal of Economic Dynamics and Control, vol. 91, pp. 104-119, 2018. Article (CrossRef Link)

[17] J. White, J. S. Park, C. A. Kamhoua, and K. A. Kwiat, "Game theoretic attack analysis in online social network services," in Proc. of the 2013 IEEE/ACM International Conference on Advances in Social Networks Analysis and Mining, pp. 1012-1019, Aug. 2013. Article (CrossRef Link)

[18] L. Richard and W. Joshua, "Analysis and results of the 1999 DARPA off-line intrusion detection evaluation," in Proc. of International Workshop on Recent Advances in Intrusion Detection, pp. 162-182, 2000. Article (CrossRef Link)

[19] A. Erwin and P. Alex, "On the stability of evolutionary dynamics in games with incomplete information," Mathematical Social Sciences, vol. 58, no. 3, pp. 310-321, 2009. Article (CrossRef Link)

[20] S. G. Hu, C. M. Huang, and F. K. Wu, Stochastic Differential Equation, Beijing: Science Press, 2008.

[21] S. Firdose, L. Lopes, W. Moreira, R. Sofia, and P. Mendes, CRAWDAD Dataset Copelabs/Usense. [Online]. Available: https://crawdad.org/copelabs/usense/20170127

[22] H. W. Zhang and T. Li, "Optimal active defense based on multi-stage attack-defense signaling game," Journal of Electronic Science, vol. 45, no. 2, pp. 431-439, 2017. Article (CrossRef Link) 


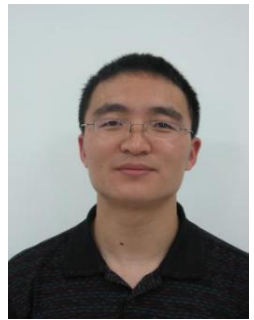

Guosheng Zhao born in 1977. Ph. D, professor, Master supervisor. Senior member of CCF. His main research interests include cognitive network, and trusted computing.

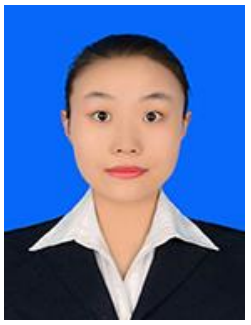

Dongmei Liu born in 1995, postgraduate. Her main research interests include crowd sensing, cognitive computing and trusted computing.

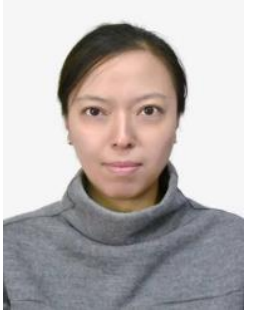

Jian Wang born in 1979. PhD, professor, Doctoral supervisor. Member of CCF. Her main research interests include crowd sensing, cognitive network, SDN and survivability. 